\title{
Von Verhalten und Verhältnissen - Gedanken zur NCD-Strategie
}

\section{Carlos Beat Quinto}

Dr. med., Mitglied des FMH-Zentralvorstandes, Departementsverantwortlicher Public Health und Gesundheitsberufe

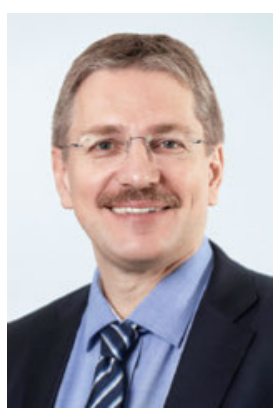

Unsere Gesundheit hängt ab von den Verhältnissen unserer natürlichen und sozialen Umwelt, wobei in der Ersteren die Qualität von Luft, Wasser und Boden und in der Letzteren Lebensstil und Verhalten subsumiert sind. Daneben tragen unsere persönlichen - nur bedingt modifizierbaren - biologischen Eigenschaften und, last, but not least, das Gesundheitswesen zur Gesundheit bei. In den nächsten Jahrzehnten werden wir rein aus demographischen Gründen mit einer $\mathrm{Zu}$ nahme nicht übertragbarer, chronischer Erkrankungen konfrontiert sein. Diese Zunahme ist durch unser heutiges Handeln mitbestimmt - und sie kommt, ob wir wollen oder nicht. Die Nationale Strategie zur Prävention nichtübertragbarer Krankheiten (NCDStrategie) wurde geboren aus der Einsicht, handeln zu müssen, und Handlungsbedarf ist gerade nach 2016 wahrlich gegeben.

Die Schweiz verfügt zwar über eines der besten Gesundheitswesen: Gemäss European Health Consumer Index gehören wir zu den Top Five. An dieser Stelle ein Dank an alle im Gesundheitswesen tätigen Berufsangehörigen, die mit ihrem persönlichen Engagement zu diesem Ergebnis beitragen. In Bezug auf die nachhaltigen, mit der Gesundheit zusammenhängenden Entwicklungsziele der Agenda 2030, zu der sich die Schweizer Politik offiziell bekennt, sieht aber unser Rang wesentlich schlechter aus: Platz 16 in Europa und Platz 19 weltweit (vgl. Lancet-Ausgabe vom 21. September 2016).

\section{Die NCD-Strategie wurde geboren aus der} Einsicht, handeln zu müssen, und Handlungsbedarf ist gerade nach 2016 wahrlich gegeben.

Dieser relativ schlechte Rang ist den Verhältnissen geschuldet, für welche letztlich die parlamentarische Arbeit verantwortlich zeichnet. Im internationalen Vergleich gibt es in der Schweiz zu viele Rauchende, Alkoholabhängige und Suizide. Alle, die in Hausarztpraxen und Notfallstationen arbeiten, wissen: Menschen mit gesundheitlichen Beschwerden infolge Tabak- und Alkoholkonsums, hyperkalorischer Ernährung, Bewegungsmangels sowie psychosozialer Belastungssituationen sind unser Alltag. Dank Parlaments- entscheiden im letzten Jahr soll sich in den nächsten 30 Jahren daran nichts ändern. Das bedeutet pro Jahr alleine infolge Tabakkonsums: gemäss gesundheitsökonomischer Abschätzung 3 bis 4 Milliarden Franken vermeidbare direkte Gesundheitskosten, das heisst Krankenkassenprämien- und Steuergelder; gemäss Regulationsfolgenabschätzung eine halbe Milliarde Franken volkswirtschaftliche Zusatzkosten und Tausende vermeidbare vorzeitige Todesfälle. Zusätzlich durch Alkohol induziertes soziales Elend, Gewalt, Unfälle und jährlich weitere Milliarden direkte Gesundheitskosten. Quoi faire?

\section{Letztlich wird sich im Umgang mit nicht} übertragbaren Erkrankungen ein integrativer Ansatz rechnen.

Positiv denken und mit den vorhandenen Mitteln arbeiten: Das heisst, unser Top-Gesundheitswesen, mit aktuell 99\% der Ausgaben, kombinieren mit der Gesundheitsförderung und Prävention, mit aktuell 1\% der Ausgaben. Letztlich wird sich ein integrativer Ansatz rechnen, auch für die Krankenkassen. Diese sollten einzig die Prämiengelder solidarisch umverteilen und gut treuhänderisch verwalten und nicht einige hundert Millionen Franken an Provisionen für Maklergeschäfte (WZW?) und Werbung zwecks Risikoselektion ausgeben. Gesundheitsförderung, Prävention, Diagnostik, Therapie, Rehabilitation und Palliation lassen sich zwar wissenschaftlich, aber im praktischen ärztlichen Alltag meist nicht trennen. Um der natürlichen Varianz gerecht zu werden, ist die Partizipation nicht nur der Patientinnen und Patienten, sondern auch aller involvierten Berufe und Fachgebiete im Gesundheitswesen angesagt. Top-down-Konzepte werden nicht goutiert. In diesem Sinne wird sich das Departement Public Health und Gesundheitsberufe in die etwas verhaltenslastige NCD-Strategie einbringen, insbesondere im Massnahmenpaket "Prävention in der Gesundheitsversorgung» (vgl. Artikel zur NCD-Strategie aus ärztlicher Sicht auf S. 100). Verhältnispräventiv werden in dieser Legislaturperiode bestenfalls gesundheitspolitische Lippenbekenntnisse zu erwarten sein. 